\title{
Structural and functional insights into the TEAD-YAP complex in the Hippo signaling pathway
}

\author{
Liming Chen ${ }^{1}$, Portia Gloria Loh ${ }^{1}$, Haiwei Song ${ }^{1,2,3 凶}$ \\ ${ }^{1}$ Cancer and Developmental Cell Biology Division, Institute of Molecular and Cell Biology, A*STAR (Agency for Science, \\ Technology and Research), 61 Biopolis Drive, Singapore 138673, Republic of Singapore \\ 2 Department of Biological Sciences, National University of Singapore, 14 Science Drive 4, Singapore 117543, Republic of \\ Singapore \\ ${ }^{3}$ School of Biological Sciences, Nanyang Technological University, 50 Nanyang Avenue, Singapore 639798, Republic of \\ Singapore
}

\ Correspondence: haiwei@imcb.a-star.edu.sg

Received November 8, 2010 Accepted November 14, 2010

\section{ABSTRACT}

The control of organ size growth is one of the most fundamental aspects of life. In the past two decades, a highly conserved Hippo signaling pathway has been identified as a key molecular mechanism for governing organ size regulation. In the middle of this pathway is a kinase cascade that negatively regulates the downstream component Yes-associated protein (YAP)/transcriptional coactivator with PDZ-binding motif (TAZ)/Yorkie through phosphorylation. Phosphorylation of YAP/TAZ/Yorkie promotes its cytoplasmic localization, leads to cell apoptosis and restricts organ size overgrowth. When the Hippo pathway is inactivated, YAP/TAZ/Yorkie translocates into the nucleus to bind to the transcription enhancer factor (TEAD/TEF) family of transcriptional factors to promote cell growth and proliferation. In this review, we will focus on the structural and functional studies on the downstream transcription factor TEAD and its coactivator YAP.

KEYWORDS Hippo, Yes-associated protein (YAP), transcription enhancer factor (TEAD), Yki, phosphorylation, crystal structure

\section{INTRODUCTION}

The size of organisms on the earth, even the size of cells within an organism, varies remarkably. Cell growth, proliferation and apoptosis together define cell size and number to control organ size (Conlon and Raff, 1999). If left unrestricted, cell proliferation can lead to uncontrolled organ size growth, which in turn results in diseases such as cancer. There are currently several pathways known to mediate the majority of cell fate decisions in metazoans, such as Notch, transforming growth factor $\beta$ (TGF- $\beta$ ), Hedgehog, Janus kinase (JAK)/ signal transducer and activator of transcription (STAT), and most recently, the Hippo pathway (for reviews, see Barolo and Posakony, 2002; Pan, 2007). The Hippo pathway was initially discovered in Drosophila through mosaic genetic screens with the key components sharing a similar overgrowth mutant phenotype (Justice et al., 1995; Xu et al., 1995; Kango-Singh et al., 2002; Tapon et al., 2002; Harvey et al., 2003; Pantalacci et al., 2003; Wu et al., 2003). The prevailing model that has emerged from genetic and biochemical studies consists of three components, namely, a set of upstream regulatory factors (Ds, Ft, Ex, Mer and Kibra), the core kinase cassette (Hpo, Sav, Wts and Mats) and the downstream transcriptional machinery (Yki and Sd) (Fig. 1). All the components are well conserved in mammals and comprise of Fat4 ( $\mathrm{Ft}$ homolog), FRMD6 (Ex homolog), NF2 (Mer homolog), Mst1/2 (Hpo homolog), WW45 (Sav homolog), Lats1/2 (Wts homolog), Mob1 (Mats homolog), YAP and TAZ (Yki homologs) and TEAD (Sd homolog) (for reviews, see Zeng and Hong, 2008; Zhao et al., 2010) (Fig. 1).

The Hippo pathway got its name from Hippo (Hpo), one of the two kinases that forms part of the core kinase module. The C-terminal domain of the STE20-like Hpo kinase associates with the N-terminal domain of its adaptor protein, Salvador (Sav), a WW-domain-containing protein, which acts as a 


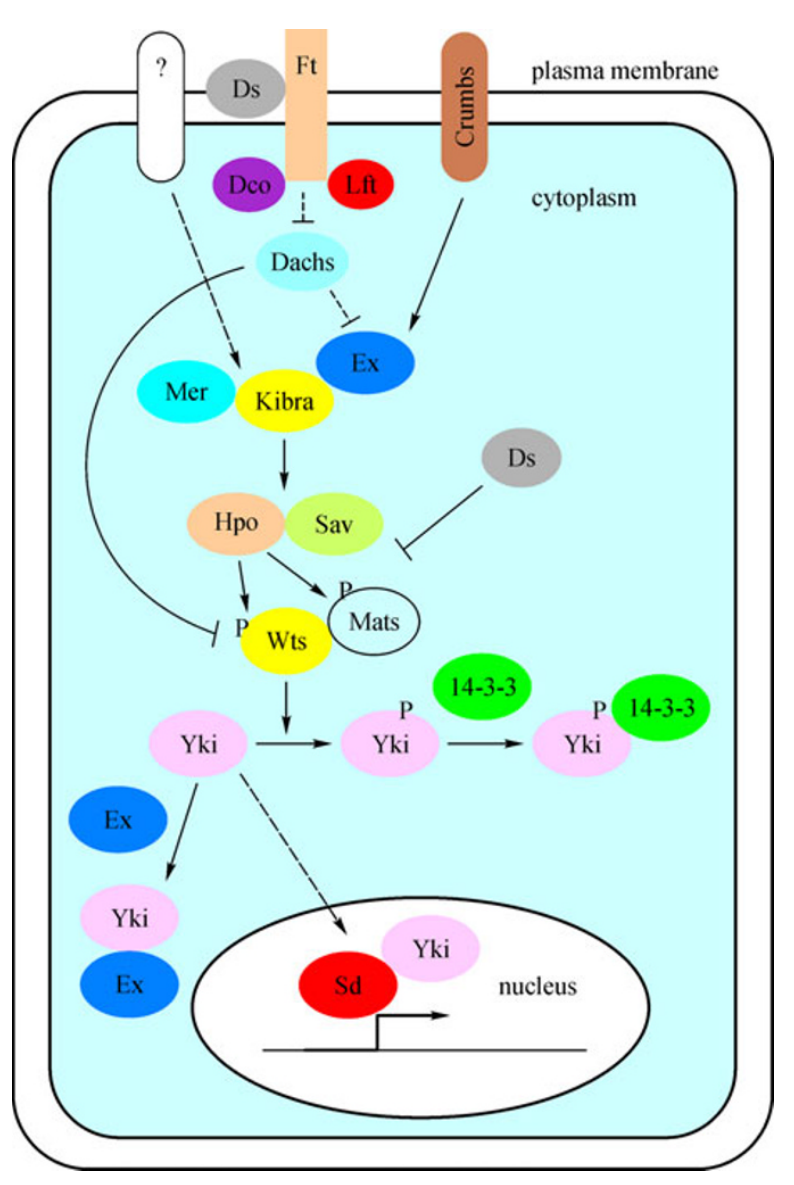

Figure 1. Working model of the Hippo pathway in Drosophila. The Hippo pathway is affected by the upstream regulators consisting of membrane proteins $\mathrm{Ft}$, Ds, and Crumbs, and the submembrane proteins Ex, Mer and Kibra. Hippo signaling involves a series of phosphorylation events mediated by the core kinase machinery consisting of Hpo, Sav, Wts and Mats, which leads to the phosphorylation of Yki and subsequent sequestration by 14-3-3 proteins, excluding it from the nucleus and its normal function of activating the transcription factor Sd. Non-phosphorylated Yki associates with Sd to form a transcription complex that will drive the expression of target genes that ultimately promote cell proliferation and cancer development. Arrowed and blunted lines indicate activation and inhibition respectively; dashed arrows indicate undetermined biochemical mechanism(s); question marks denote unknown component(s).

scaffold to assemble the entire signaling complex containing Hpo and Warts (Wts), an NRD (nuclear Dbf2-related) family protein kinase (Harvey et al., 2003; Udan et al., 2003) (Fig. 2). Upon autophosphorylation, Hpo/Sav phosphorylates Sav and Wts, resulting in Sav stabilization (Pantalacci et al., 2003; Wu et al., 2003). The coiled-coil domain of Sav, SARAH, has been implicated in Hpo binding, although the cooperative nature between the $\mathrm{N}$ terminus and SARAH domain of Sav for binding to the $\mathrm{C}$ terminus of $\mathrm{Hpo}$ and regulating its activity is still unclear (Harvey et al., 2003; Scheel and Hofmann, 2003; Wu et al., 2003) (Fig. 2). Wts also interacts directly with the two WW domains of Sav through its five PPXY motifs (Tapon et al., 2002) (Fig. 2). Additionally, Hpo phosphorylates Mats (Mob as tumor suppressor), resulting in an increased affinity for Wts and Mats in turn activates Wts to phosphorylate the downstream transcriptional coactivator Yki at three HXRXXS consensus motifs (Praskova et al., 2008). Phosphorylation of Yki at S168 creates a binding site for 14-3-3 proteins and leads to its cytoplasmic localization and subsequent inactivation (Basu et al., 2003; Dong et al., 2007; Zhao et al., 2007; Oh and Irvine, 2008; Oh et al., 2009). Phosphorylation on the S111 and S250 sites of Yki also influences Yki activity in vivo, albeit via a mechanism different from 14-3-3 sequestration and cytoplasmic retention (Oh and Irvine, 2009; Ren et al., 2010).

Yki can also be regulated by a phosphorylation-independent mode wherein Yki directly binds to Expanded (Ex) to prevent Yki from translocating into the nucleus (Badouel et al., 2009). The interaction between $\mathrm{Yki}$ and Ex is mediated by the two WW domains of Yki and three PPXY consensus motifs of Ex, all five of which are critical for efficient binding (Badouel et al., 2009; Oh et al., 2009) (Fig. 2 and 3). Furthermore, Yki phosphorylation on $\mathrm{S} 168$ is dispensable for Ex binding (Badouel et al., 2009). Ex has been demonstrated to regulate growth by directly inhibiting Yki via a reduction of the levels of Yki in the nucleus to inhibit an overgrowth phenotype, both in vitro and in vivo (Badouel et al., 2009). However it has been shown that when 14-3-3-dependent-sequestration of $Y k i$ is silenced, Yki still accumulates in the nucleus, indicating that phosphorylation-independent inhibition of $\mathrm{Yki}$ remains a minor mode of regulation.

The roles of signaling components upstream of Hpo have only recently been established. Hpo and Sav are directly regulated by a protein complex consisting of Ex, Mer and Kibra (Yu et al., 2010). Kibra contains two WW domains, the first of which mediates the interaction between Kibra and the RXPPXY motif of Ex, while the second WW domain has been postulated to be important for dimerization with Sav (Genevet et al., 2010; Yu et al., 2010) (Fig. 2). The N- and C-terminal fragments of Kibra are responsible for association with Mer (Genevet et al., 2010) (Fig. 2). The N-terminal half of Mer, which is composed mostly of the FERM domain, is required for interaction with Sav via a FERM binding consensus motif (FBM) in the $\mathrm{N}$ terminus of Sav (Yu et al., 2010) (Fig. 2). Mutation of the FBM abrogates not only Sav/Mer interaction, but also Mer-induced phosphorylation of Sav (Yu et al., 2010). Both the $\mathrm{N}$-(containing the FERM domain) and C-terminal halves of Ex can associate with $\mathrm{Hpo}$, while the SARAH domain of $\mathrm{Hpo}$ is essential for Ex interaction (Yu et al., 2010) (Fig. 2). Kibra, Mer and Ex colocalize to the apical membrane domain of epithelial cells, and function as a protein complex to regulate the Hippo signaling pathway. Two mechanisms have 


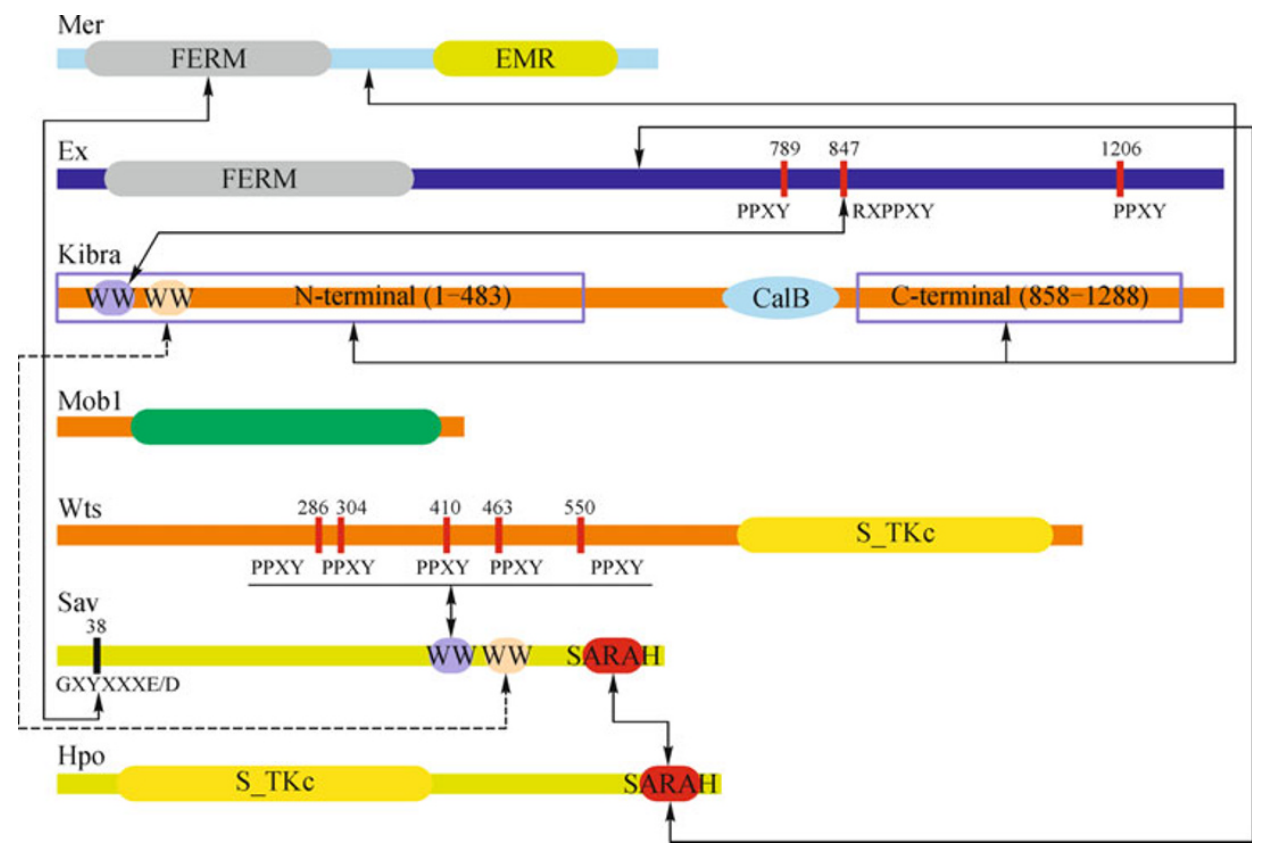

Figure 2. Schematic illustration of the domain organization and key interactions between the Hippo pathway components. The abbreviations used are as follows: FERM, F for 4.1 protein, $E$ for ezrin, $R$ for rasixin and M for moesin; EMR, ezrin, radixin and moesin; WW, 2 tryptophan residues; CalB, C2 domain; Calcium/lipid binding domain; S_TKc, serine/threonine protein kinase catalytic domain; SARAH, Sav/RASSF1/Hippo. Solid lines indicate interactions determined by biochemical studies and dotted lines indicate hypothetical interactions.

been proposed to explain the role of Fat in activation of the Hippo pathway. First, Fat, a transmembrane tumor suppressor, may be responsible for transducing growth signals to Ex in response to binding of Dachsous (Ds), thereby controlling Ex protein levels. Ft has also been found to colocalize with Ex in vivo, and hence may also be required for Ex membrane localization at apical junctions (Bennett and Harvey, 2006; Cho et al., 2006; Silva et al., 2006; Willecke et al., 2006; Tyler and Baker, 2007). Although Ds interacts with $\mathrm{Ft}$ and is regarded as the ligand of $\mathrm{Ft}$, both have been suggested to act in parallel to regulate tissue size (Willecke et al., 2006), thereby adding a further level of complexity. In the second proposed mode of regulation, Fat controls the protein levels of Wts through the myosin Dachs, which links Wts to proteins that promote proteolytic degradation of Wts (Cho et al., 2006; Feng and Irvine, 2007). Both Ft and Ds can be further modulated by binding of Lowfat ( $\mathrm{Lft}$ ) and in the case of $\mathrm{Ft}$, also regulated by phosphorylation by Discs Overgrown (Dco). In mammals, although Ft and Ex homologs (Fat4 and FRMD6) exist, their functional significance remains unclear; NF2 (Mer homolog) however retains some effect on the Hippo pathway. NF2 is not only dramatically downregulated in human mesotheliomas and gliomas, re-expression of NF2 in glioma cells inhibits cell proliferation and promotes apoptosis (Bianchi et al., 1994; Lau et al., 2008; Yokoyama et al., 2008). In particular, NF2 has been suggested to regulate the phosphorylation state of YAP and in turn, its transcriptional activity (Yokoyama et al., 2008).

\section{TEAD/TEF FAMILY TRANSCRIPTIONAL FACTORS}

The Transcription Enhancer Factor (TEAD/TEF) family of transcriptional factors is the mammalian homolog of Drosophila Sd sharing an evolutionarily conserved DNA binding TEA domain (Fig. 3A). All four TEAD members (TEAD1-4) are ubiquitously expressed in mice and humans, but their pattern of expression and downstream target genes depend on the developmental stage and cellular context (Yockey et al., 1996; Kaneko et al., 1997; Jacquemin et al., 1998; Kaneko and DePamphilis, 1998). The N-termini and prolinerich regions that are the most divergent between the four family members may account for their differences in activities. In gene knockout studies, while TEAD2 knockout mice appeared normal, TEAD1/TEAD2 double knockout mouse embryos displayed reduced cell proliferation and increased apoptosis, and experienced lethality around embryonic day 9.5 with severe growth defects and morphological abnormalities, suggesting that TEAD1 plays a more important proliferative role in development (Sawada et al., 2008). An earlier genetic study provided direct evidence for the involvement of TEAD1 in human disease. A missense mutation $(\mathrm{Y} 421 \mathrm{H})$ of TEAD1 was observed in sufferers of 
A

\begin{tabular}{|c|c|c|c|c|c|}
\hline TEAD & TEA & \multicolumn{4}{|c|}{ YAP binding } \\
\hline YAP & $\mathrm{TE}$ & WW & WW & $\overline{C C}$ & transactivation domain \\
\hline
\end{tabular}
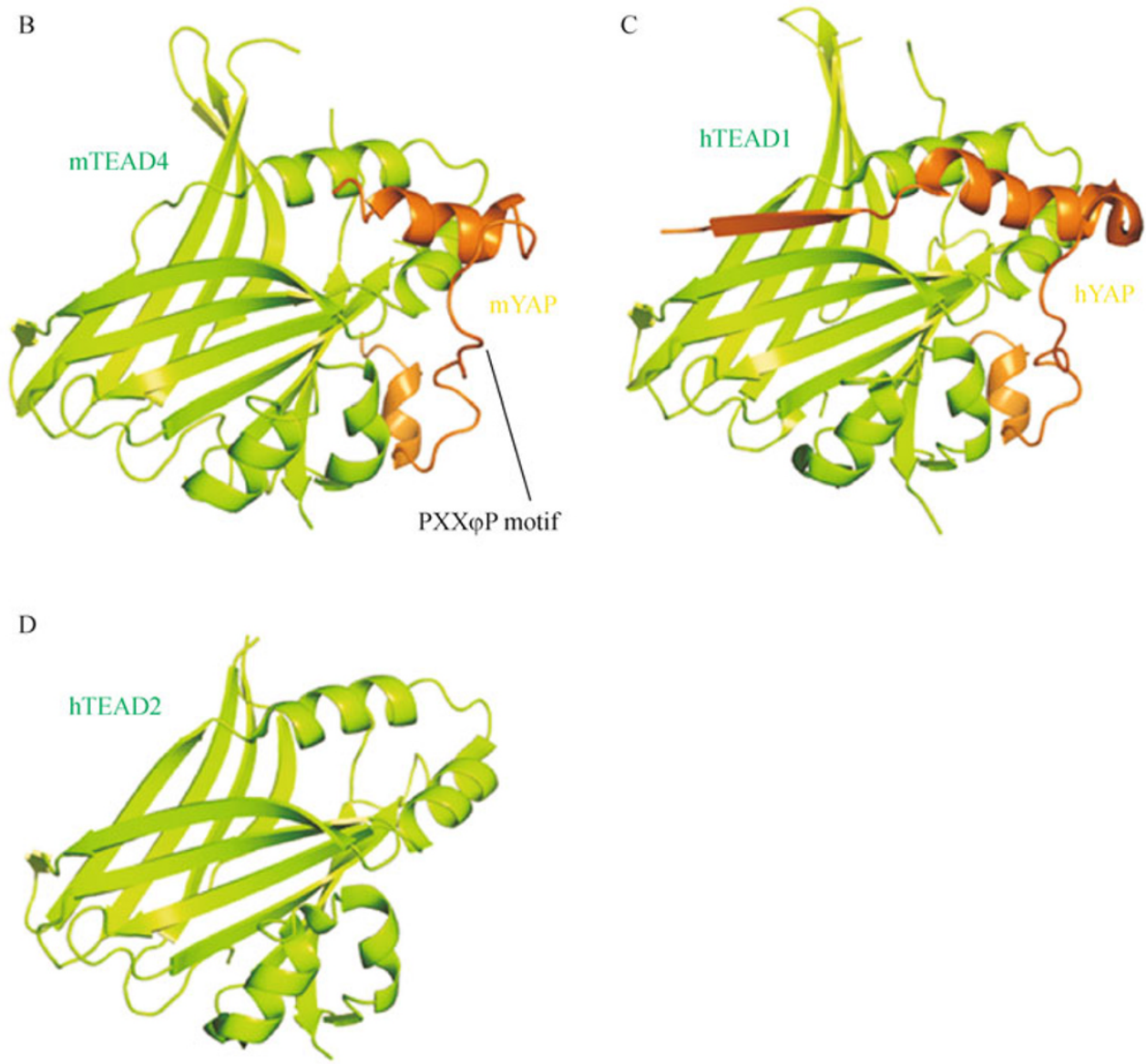

Figure 3. Structural comparison between mTEAD4-YAP, hTEAD1-YAP and hTEAD2. (A) Schematic illustration of the domain organization of TEAD and YAP showing the TEA domain (pink), YAP binding domain (green), proline-rich region (yellow), TEAD binding domain (orange), WW domains (blue), SH3 binding domain (brown), coiled-coil (CC) domain in hot pink, transcription activation domain (pale yellow) and PDZ binding domain (dark blue). The abbreviations used are as follows: TEA, transcription enhancer factor; YAP, Yes-associated protein; Pro-rich, Proline-rich region; SH3, SRC homology 3 domain; PDZ, post synaptic density protein (PSD95), Drosophila disc large tumor suppressor (DlgA), zonula occludens-1 protein (zo-1). (B) Crystal structure of mouse TEAD4 (green) complexed with mouse YAP (orange) (Protein Data Bank (PDB) accession code 3JUA) (Chen et al., 2010). The PXX $\phi P$ motif is highlighted. (C) Crystal structure of human TEAD1 (green) complexed with human YAP (orange) (PDB accession code 3KYS) (Li et al., 2010). (D) Crystal structure of human TEAD2 (green) (PDB accession code 3L15) (Tian et al., 2010).

human Sveinsson's chorioretinal atrophy (SCRA), an autosomal dominant eye disease characterized by symmetric lesions radiating from the optic disc involving the retina and choroid (Fossdal et al., 2004). This conserved residue was found to be defective in YAP and TAZ binding when mutated (Kitagawa, 2007). Recent biochemical and genetic studies have established TEAD as a key downstream mediator of YAP (mammalian homolog of Yki) activity and distribution in Hippo signaling (Ota and Sasaki, 2008; Zhao et al., 2008; Zhang et al., 2009). TEAD1 has also been implicated in prostate and pancreatic cancer (Hucl et al., 2007; Kwon et al., 2007; Knight et al., 2008). 
It has recently emerged that TEAD4 contributes to the fate of cell lineage specification by regulating trophectoderm function, but not inner cell mass specification (Yagi et al., 2007; Nishioka et al., 2008). The Hippo pathway was shown to regulate differential signaling between the outside and inside cells of the preimplantation mouse embryo. In the outside cells, the Hippo kinase module is inactivated, allowing YAP to localize to the nuclei and activate TEAD4 transcription. Whereas in the inside cells, YAP is phosphorylated by Lats and sequestered in the cytoplasm (Nishioka et al., 2009). TEAD4 transcript levels have also been found to be amplified in breast (Richardson et al., 2006; Adélaïde et al., 2007; Han et al., 2008), testicular (Almstrup et al., 2004; Skotheim et al., 2006), brain (Weber et al., 1996; Ehrbrecht et al., 2006), ovarian (Nowee et al., 2007), throat (Rodriguez et al., 2005), and head and neck (Singh et al., 2001) cancers. Soft agar assays further support TEAD4's possible role as an oncogenic protein during cancer development. TEAD4 overexpression resulted in an increase in transformation ability of MCF10A and NIH3T3 cells (Chan et al., 2009; Chen et al., 2010).

\section{YAP AND TAZ TRANSCRIPTIONAL CO-ACTIVATORS}

YAP is the mammalian homolog of Yki and has been found to be able to functionally rescue Yki mutations in Drosophila (Huang et al., 2005). The domain organization of YAP consists of an $\mathrm{N}$-terminal proline-rich region, a TEAD binding domain, two tandem WW domains, an SH3 binding motif, a coiled-coil domain, a transcription activation domain and a $\mathrm{C}$ terminal PDZ binding motif (Fig. 3A). YAP is a potent growth promoter and overexpression of YAP increases organ size in Drosophila and saturation cell density of NIH3T3 cells (Zhao et al., 2007). Several lines of evidence have indicated that YAP is a candidate oncogenic protein in mammalian cells. First, yap has been observed in the human chromosome 11q22 amplicon, a hallmark of several human cancers (Overholtzer et al., 2006; Zender et al., 2006). Secondly, YAP is overexpressed and has increased nuclear localization in multiple types of human tumors, including colonic, lung, ovarian, hepatocellular, prostate, pancreatic, gastric and oral carcinomas (Baldwin et al., 2005; Snijders et al., 2005; LamHimlin et al., 2006; Modena et al., 2006; Dong et al., 2007; Zhao et al., 2007; Steinhardt et al., 2008; Yokoyama et al., 2008; Wang et al., 2010). Third, overexpression of YAP in immortalized epithelial MCF10A cells induced epithelialmesenchymal transition (EMT), which is often associated with cancer stem cell property and cancer metastasis (Overholtzer et al., 2006; Polyak and Weinberg, 2009). Finally, YAP was found to cooperate with Myc oncogene to stimulate tumor growth in nude mice (Zender et al., 2006). Further support for YAP being an oncogenic protein comes from observations that transgenic mice with liver-specific YAP overexpression showed a dramatic increase in liver size and eventually developed tumors (Camargo et al., 2007; Dong et al., 2007).

The paralog of YAP, TAZ, was originally identified as a 143-3 binding protein (Kanai et al., 2000). It has a similar domain structure to YAP, with the exception of the proline-rich region, the second WW domain and the $\mathrm{SH} 3$ binding motif. Similar to YAP, TAZ induces EMT, increases cell migration and invasion by mediating TEAD transcriptional activity (Chan et al., 2008; Lei et al., 2008). A recent study demonstrates that TAZ regulates expression of the EMT transcription factor zinc finger E-box binding homeobox 1 (ZEB1) to control retinal pigment epithelial cell proliferation and differentiation by binding to the ZEB1 promoter (Liu et al., 2010). Consequently, translocation of TAZ-TEAD1 complex into the nucleus was associated with a loss of intercellular contact, while knockdown of TAZ prevented proliferation and EMT. There is also evidence that TAZ may function as a co-activator of the MyoD transcription factor to promote expression of MyoD target genes to enhance myogenic differentiation (Jeong et al., 2010). In addition, a link between TAZ and Wnt signaling was discovered recently when it was shown that the Hippo pathway is able to restrict $W n t / \beta$-catenin signaling by promoting an interaction between TAZ and Disheveled (DVL) in the cytoplasm (Varelas et al., 2010). Interestingly, YAP and TAZ have also been implicated in the maintenance of stem cell pluripotency. An earlier seminal work by Yaffe and colleagues demonstrated the role of TAZ as a regulator of mesenchymal stem cell differentiation by promoting osteoblast differentiation, which is dependent on Runx2-mediated gene transcription (Hong et al., 2005). Recent data indicate that TAZ is essential to maintain the expression of pluripotency markers Oct4 and Nanog in human embryonic stem cells by regulating Smad nuclear shuttling (Varelas et al., 2010). Further support for the notion that the Hippo signaling pathway is linked to pluripotency maintenance comes from the evidence that YAP mediates bone morphogenetic protein (BMP) target gene expression in mouse embryonic stem cells (Alarcon et al., 2009).

TAZ is regulated in a similar way to YAP. While YAP is inactivated through Lats phosphorylation at serine 127 and subsequent cytoplasmic retention (Zhao et al., 2007; Hao et al., 2008), TAZ undergoes phosphorylation at serine 89 (Lei et al., 2008). Mutagenesis of this residue to alanine greatly enhances the oncogenic behavior of TAZ. Conversely, mutations of TAZ residues involved in TEAD binding abrogated TAZ-mediated transformation, indicating that TEADs modulate the ability of TAZ to promote cell proliferation, EMT and oncogenesis (Chan et al., 2009; Zhang et al., 2009; Chen et al., 2010). Furthermore, the observations that YAP and TAZ knockout mice show different phenotypes suggest that YAP and TAZ do not compensate each other and may have distinct regulatory events in cellular and/or developmental processes (Morin-Kensicki et al. 2006; Hossain et al. 2007; Makita et al. 2008). 


\section{THE INTERACTION OF TEAD/TEF WITH YKI/YAP MEDIATES THE OUTPUT OF THE HIPPO PATHWAY}

While it was known that Yki/YAP functions as a transcriptional co-activator since it lacks a DNA binding domain (Yagi et al., 1999), the identity of its target transcription factor remained enigmatic. Several transcription factors were found to interact with Yki/YAP such as RUNX1/2 (Yagi et al., 1999), ErbB4 (Komuro et al., 2003), and p73 (Strano et al., 2001; Omerovic et al., 2004); but none was found to be functionally linked to the Hippo pathway. Although TEAD/TEF family transcription factors were identified as Yki/YAP partners as early as 2001 (Vassilev et al., 2001), the functional link between the Hippo signaling pathway and the interaction of TEAD/TEF family transcription factors and Yki/YAP was established only recently in Drosophila and mammals (Goulev et al., 2008; Wu et al., 2008; Zhang et al., 2008). In Drosophila, the TEAD/ TEF transcription factor Scalloped (Sd) mediates Hippo signaling in organ size control by functioning as a Yki partner. This interaction has been demonstrated both in vitro and in vivo (Goulev et al., 2008; Wu et al., 2008; Zhang et al., 2008). Inactivation of Sd shows similar phenotypes to a loss of Yki which is characterized by a suppression of tissue overgrowth and target gene expression caused by tumor suppressor mutations or Yki overexpression in the Hippo pathway, while an overexpression of $\mathrm{Sd}$ enhances tissue overgrowth (Wu et al., 2008; Zhang et al., 2008; Zhao et al., 2008). Further evidence that supports the link between $\mathrm{Yki}$ and $\mathrm{Sd}$ comes from a Yki missense mutation that abrogates Sd-Yki binding as well as inactivates Yki function in vivo (Wu et al., 2008). In mammalian cells, YAP is found to bind strongly to four TEAD homolog proteins (TEAD 1-4) both in vitro and in vivo, and this interaction mediates YAP-dependent gene induction and growth control in human and mouse cells (Sawada et al., 2008; Zhao et al., 2008). Furthermore, TEAD1/2 double knockout mice are lethal and manifest proliferation defects similar to that of YAP knockout mice (Sawada et al., 2008). Knockdown of TEAD1/3/4 also markedly shrank the enlarged acini caused by YAP-5SA expression (Zhao et al., 2008). Further compelling evidence demonstrated that the YAPS94A mutant, which lost its ability to physically interact with TEADs, abolishes its ability to activate TEADs for YAPinduced overgrowth, EMT and oncogenic transformation in MCF10A cells (Zhao et al., 2008).

Although the functional conservation between Drosophila Sd-Yki complex and mammalian TEADs-YAP has been determined (Wu et al., 2008; Zhao et al., 2008), the genes regulated by TEADs-YAP in mammals are different from those regulated by Sd-Yki. Cyclin E and IAP, which are the key Yki-inducible genes in Drosophila, were found to be not significantly induced by YAP in either mouse (i.e., NIH-3T3) or human cells (i.e., MCF10A) (Zhao et al., 2008). This finding might indicate that although the key components of the Hippo pathway are conserved between Drosophila and mammals, the detailed mechanism between both species might have substantial differences.

\section{STRUCTURAL STUDIES OF TEAD AND THE TEAD-YAP COMPLEX}

TEAD/TEF transcriptional factors interact with the $\mathrm{N}$-terminal of Yki/YAP via their C-terminals. Two recently determined structures clarified how TEAD mediates the function of YAPinduced gene expression (Chen et al., 2010; Li et al., 2010) (Fig. 3B and $3 \mathrm{C}$ ). The first is the crystal structure of the Cterminal of mouse TEAD4 in complex with the $\mathrm{N}$-terminal of mouse YAP (mouse TEAD4-YAP) (Fig. 3B), and the other is the crystal structure of the C-terminal of human TEAD1 in complex with the $\mathrm{N}$-terminal of human YAP (human TEAD1YAP) (Fig. 3C). In the structure of mouse TEAD4-YAP complex, TEAD4 adopts an immunoglobulin-like $\beta$-sandwich fold flanked by four short $\alpha$ helices, while YAP resembles a belt wrapped around TEAD4 via folding into two a helices linked by a $\mathrm{PXX} \phi \mathrm{P}$ motif-containing loop. The structure of human TEAD1-YAP, as expected, is highly similar to the structure of mouse TEAD4-YAP. However, YAP in the human complex contains an additional $\mathrm{N}$-terminal $\beta$ strand and thus presents an extra recognition interface with the immunoglobulinlike $\beta$-sandwich fold in TEAD1. It is not surprising that deletion of this additional $\mathrm{N}$-terminal $\beta$ strand in hYAP had little effect on its interaction with human TEAD1 (Li et al., 2010) as a synthetic human YAP peptide encompassing both the a helices (amino acids 61-100) has been shown to be sufficient for binding to TEAD (Tian et al., 2010). Structural comparison of the TEAD-YAP complex with the C-terminal fragment of human TEAD2 (Tian et al., 2010) revealed a lack of conformational rearrangements in TEAD upon binding to YAP (r.m.s.d. 0.75) (Fig. 3D). The C-terminal of TEADs presented a rigid interface for specific interaction with YAP, while nuclear magnetic resonance (NMR) studies have revealed that the $\mathrm{N}$-terminal TEAD binding domain of YAP exists in an unfolded conformation in its native state and that TEAD binding causes localized conformational changes in YAP (Tian et al., 2010). More importantly, point mutation studies of TEAD4 based on the mouse TEAD4-YAP complex structure indicate that the residues important for YAP interaction are required for TEAD4 transforming activity on soft agar assay. Although the mouse TEAD4-YAP complex structure is highly similar to the structure of human TEAD1YAP (r.m.s.d. 0.71), both structures have provided somewhat conflicting suggestions on the importance of a PXX $\phi P$ motif, a loop conserved in YAP and the mammalian homolog Yki but not in TAZ. While it has emerged from the report by Chen et al. that this motif not only plays a pivotal role in the interaction with TEAD4, but also is crucial for YAP's transforming ability (Chen et al., 2010). Li et al. have downplayed this suggestion, indicating that the PXX $\phi P$ motif contributes a minor role in binding TEAD (Li et al., 2010). Although whether the PXX $\phi P$ 
motif is a bona fide TEAD binding site remains to be rigorously tested, both structures have elucidated the structural basis for TEAD/YAP interaction.

Importantly, both structures have shed light on the etiology of SCRA. Apart from identifying the residues important for TEAD/YAP interaction through point mutations, these structures have explained the essential role of a tyrosine residue (Y422 in mTEAD4 and Y406 in hTEAD1), whose nonconservative missense mutation to a histidine has been implicated in this genetic disease (Fossdal et al., 2004). This tyrosine was found to be involved in hydrogen bond interactions with a corresponding serine residue in YAP (S79 in mYAP and S94 in hYAP (Chen et al., 2010; Li et al., 2010). Mutation of this tyrosine residue in TEAD1 abolished its interaction with YAP and TAZ (Kitagawa, 2007). Substitution of this residue by His or any other residue would disrupt its interaction with YAP and account for the observed biochemical and pathological phenotypes.

Given that the Hippo pathway is implicated in cancer and YAP is regarded as an oncogenic protein, the structures of TEAD and YAP may provide a new strategy for cancer therapeutics by disrupting the TEAD-YAP interaction. Both structures show that only a small fragment in the $\mathrm{N}$-terminal region of YAP is involved in the interaction of TEAD, and that all the three important binding regions identified by Chen et al. (the two $\alpha$ helices and the PXX $\phi P$ motif containing linker region) are required for interaction between YAP and TEAD and are hence crucial specificity determinants (unpublished data). Targeting the YAP binding surface on TEAD by either designing a peptide molecule that mimics the region of YAP bound to TEAD in the structure or a small molecule that selectively blocks any of the three important binding regions between YAP and TEAD might be an effective strategy to abolish the TEAD-YAP interaction and may have anticancer therapeutic potential.

\section{FUTURE PERSPECTIVES}

The past several years have witnessed rapid progress in Hippo pathway research. These advances made mainly by genetic and biochemical studies have greatly increased our understanding of the molecular mechanisms underlying the Hippo signaling pathway. Complementary to these studies, structural biology has been emerging as a key approach to reveal mechanistic insights into Hippo signaling. In addition to the structures of TEAD in isolation and in complex with YAP, the structures of several components of the Hippo pathway have been determined. These include Mats/Mob1 (PDB ID: 1PI1) (Stavridi et al., 2003), Mst1 (PDB ID: 3COM), the Sav WW domain (PDB ID: 2YSB, 2DWV) (Ohnishi et al., 2007), Merlin's FERM domain (PDB ID: 1ISN) (Shimizu et al., 2002), the $\mathrm{C} 2$ domain of KIBRA (PDB ID: 2ZOU), and the UBA domain from mouse LATS2 (PDB ID: 2COS). Altogether these structures have extended our understanding of the molecular mechanism governing the Hippo pathway. However, many key questions remain to be addressed. The major future challenge is to identify the molecular nature of the upstream signals and to elucidate how these signals regulate the Kibra-Ex-Mer complex and trigger the core kinase cassette. Structural studies on the Kibra-Ex-Mer complex and its interaction with $\mathrm{Hpo} / \mathrm{Sav}$ are required to reveal the mechanism underlying these processes.

Another outstanding question in the outputs of the pathway is how YAP coordinates its binding to TEAD with the binding of canonical M-CAT elements to TEAD to regulate target gene expression. M-CAT sequence motifs (5'-TCATTCCT-3') are found in several muscle-specific gene promoters and are important for the regulation of these genes during embryonic development and disease (Larkin et al., 1996; Jiang et al., 2000). TEADs contain an $\mathrm{N}$-terminal TEA domain, which is a DNA binding module, and a C-terminal protein binding domain, which interacts with the N-terminal of YAP. The solution structure of the $\mathrm{N}$-terminal TEA domain of TEAD has provided a model for how TEAD uses its N-terminal TEA domain to bind M-CAT elements (Anbanandam et al., 2006), and the two TEAD-YAP complex structures explain how TEADs and YAP interact with each other (Chen et al., 2010; $\mathrm{Li}$ et al., 2010). Although the $\mathrm{N}$-terminal of YAP has been shown to undergo a significant conformational change from its native unfolded form upon the binding of the C-terminal of TEAD, TEAD, on the other hand, has no conformational change occurring upon binding to YAP. It remains unknown how the full-length TEAD protein uses its two functional domains (TEA domain and YAP-binding domain) for binding to YAP and the M-CAT element simultaneously. The complex structure of the heterotrimeric TEAD, YAP and M-CAT motif would reveal the mechanism by which YAP modulates TEAD transcription activity to regulate target gene expression to control the output of the Hippo pathway. Finally, given that increased TAZ/YAP-TEAD activity is associated with diverse human cancers, inhibiting YAP/TAZ may offer a potential therapeutic strategy for treatment of human cancers.

\section{ABBREVIATIONS}

Ds, dachsous; Ex, expanded; FERM, F for 4.1 protein $E$ for ezrin, $R$ for rasixin and $M$ for moesin.Ft; fat; Hpo, Hippo; Lats1, large tumor suppressor 1; Mats1, Mob as tumor suppressor; Mer, merlin; Sav, salvador; Sd, scalloped; TAZ, Transcriptional coactivator with PDZbinding motif; TEAD/TEF, transcription enhancer factor; Wts, warts; YAP, Yes-associated protein; Yki, Yorkie

\section{REFERENCES}

Adélaïde, J., Finetti, P., Bekhouche, I., Repellini, L., Geneix, J., Sircoulomb, F., Charafe-Jauffret, E., Cervera, N., Desplans, J., Parzy, D., et al. (2007). Integrated profiling of basal and luminal breast cancers. Cancer Res 67, 11565-11575.

Alarcón, C., Zaromytidou, A.I., Xi, Q., Gao, S., Yu, J., Fujisawa, S., 
Barlas, A., Miller, A.N., Manova-Todorova, K., Macias, M.J., et al. (2009). Nuclear CDKs drive Smad transcriptional activation and turnover in BMP and TGF-beta pathways. Cell 139, 757-769.

Almstrup, K., Hoei-Hansen, C.E., Wirkner, U., Blake, J., Schwager, C., Ansorge, W., Nielsen, J.E., Skakkebaek, N.E., Rajpert-De Meyts, E., and Leffers, H. (2004). Embryonic stem cell-like features of testicular carcinoma in situ revealed by genome-wide gene expression profiling. Cancer Res 64, 4736-4743.

Anbanandam, A., Albarado, D.C., Nguyen, C.T., Halder, G., Gao, X., and Veeraraghavan, S. (2006). Insights into transcription enhancer factor 1 (TEF-1) activity from the solution structure of the TEA domain. Proc Natl Acad Sci U S A 103, 17225-17230.

Badouel, C., Gardano, L., Amin, N., Garg, A., Rosenfeld, R., Le Bihan, T., and McNeill, H. (2009). The FERM-domain protein Expanded regulates Hippo pathway activity via direct interactions with the transcriptional activator Yorkie. Dev Cell 16, 411-420.

Baldwin, C., Garnis, C., Zhang, L., Rosin, M.P., and Lam, W.L. (2005). Multiple microalterations detected at high frequency in oral cancer. Cancer Res 65, 7561-7567.

Barolo, S., and Posakony, J.W. (2002). Three habits of highly effective signaling pathways: principles of transcriptional control by developmental cell signaling. Genes Dev 16, 1167-1181.

Basu, S., Totty, N.F., Irwin, M.S., Sudol, M., and Downward, J. (2003). Akt phosphorylates the Yes-associated protein, YAP, to induce interaction with 14-3-3 and attenuation of p73-mediated apoptosis. Mol Cell 11, 11-23.

Bennett, F.C., and Harvey, K.F. (2006). Fat cadherin modulates organ size in Drosophila via the Salvador/Warts/Hippo signaling pathway. Curr Biol 16, 2101-2110.

Bianchi, A.B., Hara, T., Ramesh, V., Gao, J., Klein-Szanto, A.J., Morin, F., Menon, A.G., Trofatter, J.A., Gusella, J.F., Seizinger, B. R., et al. (1994). Mutations in transcript isoforms of the neurofibromatosis 2 gene in multiple human tumour types. Nat Genet 6, 185-192.

Camargo, F.D., Gokhale, S., Johnnidis, J.B., Fu, D., Bell, G.W., Jaenisch, R., and Brummelkamp, T.R. (2007). YAP1 increases organ size and expands undifferentiated progenitor cells. Curr Biol 17, 2054-2060.

Chan, S.W., Lim, C.J., Guo, K., Ng, C.P., Lee, I., Hunziker, W., Zeng, Q., and Hong, W. (2008). A role for TAZ in migration, invasion, and tumorigenesis of breast cancer cells. Cancer Res 68, 2592-2598.

Chan, S.W., Lim, C.J., Loo, L.S., Chong, Y.F., Huang, C., and Hong, W. (2009). TEADs mediate nuclear retention of TAZ to promote oncogenic transformation. J Biol Chem 284, 14347-14358.

Chen, L., Chan, S.W., Zhang, X., Walsh, M., Lim, C.J., Hong, W., and Song, H. (2010). Structural basis of YAP recognition by TEAD4 in the hippo pathway. Genes Dev 24, 290-300.

Cho, E., Feng, Y., Rauskolb, C., Maitra, S., Fehon, R., and Irvine, K.D. (2006). Delineation of a Fat tumor suppressor pathway. Nat Genet $38,1142-1150$.

Conlon, I., and Raff, M. (1999). Size control in animal development. Cell 96, 235-244.

Dong, J., Feldmann, G., Huang, J., Wu, S., Zhang, N., Comerford, S. A., Gayyed, M.F., Anders, R.A., Maitra, A., and Pan, D. (2007). Elucidation of a universal size-control mechanism in Drosophila and mammals. Cell 130, 1120-1133.

Ehrbrecht, A., Müller, U., Wolter, M., Hoischen, A., Koch, A., Radlwimmer, B., Actor, B., Mincheva, A., Pietsch, T., Lichter, P., et al. (2006). Comprehensive genomic analysis of desmoplastic medulloblastomas: identification of novel amplified genes and separate evaluation of the different histological components. J Pathol 208, 554-563.

Feng, Y., and Irvine, K.D. (2007). Fat and expanded act in parallel to regulate growth through warts. Proc Natl Acad Sci U S A 104, 20362-20367.

Fossdal, R., Jonasson, F., Kristjansdottir, G.T., Kong, A., Stefansson, H., Gosh, S., Gulcher, J.R., and Stefansson, K. (2004). A novel TEAD1 mutation is the causative allele in Sveinsson's chorioretinal atrophy (helicoid peripapillary chorioretinal degeneration). Hum Mol Genet 13, 975-981.

Genevet, A., Wehr, M.C., Brain, R., Thompson, B.J., and Tapon, N. (2010). Kibra is a regulator of the Salvador/Warts/Hippo signaling network. Dev Cell 18, 300-308.

Goulev, Y., Fauny, J.D., Gonzalez-Marti, B., Flagiello, D., Silber, J., and Zider, A. (2008). SCALLOPED interacts with YORKIE, the nuclear effector of the hippo tumor-suppressor pathway in Drosophila. Curr Biol 18, 435-441.

Han, W., Jung, E.M., Cho, J., Lee, J.W., Hwang, K.T., Yang, S.J., Kang, J.J., Bae, J.Y., Jeon, Y.K., Park, I.A., et al. (2008). DNA copy number alterations and expression of relevant genes in triplenegative breast cancer. Genes Chromosomes Cancer 47, 490-499.

Hao, Y., Chun, A., Cheung, K., Rashidi, B., and Yang, X. (2008). Tumor suppressor LATS1 is a negative regulator of oncogene YAP. J Biol Chem 283, 5496-5509.

Harvey, K.F., Pfleger, C.M., and Hariharan, I.K. (2003). The Drosophila Mst ortholog, hippo, restricts growth and cell proliferation and promotes apoptosis. Cell 114, 457-467.

Hong, J.H., Hwang, E.S., McManus, M.T., Amsterdam, A., Tian, Y., Kalmukova, R., Mueller, E., Benjamin, T., Spiegelman, B.M., Sharp, P.A., et al. (2005). TAZ, a transcriptional modulator of mesenchymal stem cell differentiation. Science 309, 1074-1078.

Hossain, Z., Ali, S.M., Ko, H.L., Xu, J., Ng, C.P., Guo, K., Qi, Z., Ponniah, S., Hong, W., and Hunziker, W. (2007). Glomerulocystic kidney disease in mice with a targeted inactivation of Wwtr1. Proc Natl Acad Sci U S A 104, 1631-1636.

Huang, J., Wu, S., Barrera, J., Matthews, K., and Pan, D. (2005). The Hippo signaling pathway coordinately regulates cell proliferation and apoptosis by inactivating Yorkie, the Drosophila Homolog of YAP. Cell 122, 421-434.

Hucl, T., Brody, J.R., Gallmeier, E., lacobuzio-Donahue, C.A., Farrance, I.K., and Kern, S.E. (2007). High cancer-specific expression of mesothelin (MSLN) is attributable to an upstream enhancer containing a transcription enhancer factor dependent MCAT motif. Cancer Res 67, 9055-9065.

Jacquemin, P., Sapin, V., Alsat, E., Evain-Brion, D., Dollé, P., and Davidson, I. (1998). Differential expression of the TEF family of transcription factors in the murine placenta and during differentiation of primary human trophoblasts in vitro. Dev Dyn 212, 423-436.

Jeong, H., Bae, S., An, S.Y., Byun, M.R., Hwang, J.H., Yaffe, M.B., Hong, J.H., and Hwang, E.S. (2010). TAZ as a novel enhancer of MyoD-mediated myogenic differentiation. FASEB J 24, 3310-3320.

Jiang, S.W., Desai, D., Khan, S., and Eberhardt, N.L. (2000). Cooperative binding of TEF-1 to repeated GGAATG-related consensus elements with restricted spatial separation and 
orientation. DNA Cell Biol 19, 507-514.

Justice, R.W., Zilian, O., Woods, D.F., Noll, M., and Bryant, P.J. (1995). The Drosophila tumor suppressor gene warts encodes a homolog of human myotonic dystrophy kinase and is required for the control of cell shape and proliferation. Genes Dev 9, 534-546.

Kanai, F., Marignani, P.A., Sarbassova, D., Yagi, R., Hall, R.A., Donowitz, M., Hisaminato, A., Fujiwara, T., Ito, Y., Cantley, L.C., et al. (2000). TAZ: a novel transcriptional co-activator regulated by interactions with 14-3-3 and PDZ domain proteins. EMBO J 19, 6778-6791.

Kaneko, K.J., Cullinan, E.B., Latham, K.E., and DePamphilis, M.L. (1997). Transcription factor mTEAD-2 is selectively expressed at the beginning of zygotic gene expression in the mouse. Development 124, 1963-1973.

Kaneko, K.J., and DePamphilis, M.L. (1998). Regulation of gene expression at the beginning of mammalian development and the TEAD family of transcription factors. Dev Genet 22, 43-55.

Kango-Singh, M., Nolo, R., Tao, C., Verstreken, P., Hiesinger, P.R., Bellen, H.J., and Halder, G. (2002). Shar-pei mediates cell proliferation arrest during imaginal disc growth in Drosophila. Development 129, 5719-5730.

Kitagawa, M. (2007). A Sveinsson's chorioretinal atrophy-associated missense mutation in mouse Tead1 affects its interaction with the co-factors YAP and TAZ. Biochem Biophys Res Commun 361, 1022-1026.

Knight, J.F., Shepherd, C.J., Rizzo, S., Brewer, D., Jhavar, S., Dodson, A.R., Cooper, C.S., Eeles, R., Falconer, A., Kovacs, G., et al. (2008). TEAD1 and c-Cbl are novel prostate basal cell markers that correlate with poor clinical outcome in prostate cancer. $\mathrm{Br} \mathrm{J}$ Cancer 99, 1849-1858.

Komuro, A., Nagai, M., Navin, N.E., and Sudol, M. (2003). WW domain-containing protein YAP associates with ErbB-4 and acts as a co-transcriptional activator for the carboxyl-terminal fragment of ErbB-4 that translocates to the nucleus. J Biol Chem 278, 33334-33341.

Kwon, S., Munroe, X., Crawley, S.C., Lee, H.Y., Spong, S., Bradham, D., Gum, J.R. Jr, Sleisenger, M.H., and Kim, Y.S. (2007). Expression of connective tissue growth factor in pancreatic cancer cell lines. Int J Oncol 31, 693-703.

Lam-Himlin, D.M., Daniels, J.A., Gayyed, M.F., Dong, J., Maitra, A., Pan, D., Montgomery, E.A., and Anders, R.A. (2006). The hippo pathway in human upper gastrointestinal dysplasia and carcinoma: a novel oncogenic pathway. Int J Gastrointest Cancer 37, 103-109.

Larkin, S.B., Farrance, I.K., and Ordahl, C.P. (1996). Flanking sequences modulate the cell specificity of M-CAT elements. Mol Cell Biol 16, 3742-3755.

Lau, Y.K., Murray, L.B., Houshmandi, S.S., Xu, Y., Gutmann, D.H., and $Y u, Q$. (2008). Merlin is a potent inhibitor of glioma growth. Cancer Res 68, 5733-5742.

Lei, Q.Y., Zhang, H., Zhao, B., Zha, Z.Y., Bai, F., Pei, X.H., Zhao, S., Xiong, Y., and Guan, K.L. (2008). TAZ promotes cell proliferation and epithelial-mesenchymal transition and is inhibited by the hippo pathway. Mol Cell Biol 28, 2426-2436.

Li, Z., Zhao, B., Wang, P., Chen, F., Dong, Z., Yang, H., Guan, K.L., and $\mathrm{Xu}, \mathrm{Y}$. (2010). Structural insights into the YAP and TEAD complex. Genes Dev 24, 235-240.

Liu, Y., Xin, Y., Ye, F., Wang, W., Lu, Q., Kaplan, H.J., and Dean, D.C. (2010). Taz-tead1 links cell-cell contact to zeb1 expression, proliferation, and dedifferentiation in retinal pigment epithelial cells. Invest Ophthalmol Vis Sci 51, 3372-3378.

Makita, R., Uchijima, Y., Nishiyama, K., Amano, T., Chen, Q., Takeuchi, T., Mitani, A., Nagase, T., Yatomi, Y., Aburatani, H., et al. (2008). Multiple renal cysts, urinary concentration defects, and pulmonary emphysematous changes in mice lacking TAZ. Am J Physiol Renal Physiol 294, F542-F553.

Modena, P., Lualdi, E., Facchinetti, F., Veltman, J., Reid, J.F., Minardi, S., Janssen, I., Giangaspero, F., Forni, M., Finocchiaro, G., et al. (2006). Identification of tumor-specific molecular signatures in intracranial ependymoma and association with clinical characteristics. J Clin Oncol 24, 5223-5233.

Morin-Kensicki, E.M., Boone, B.N., Howell, M., Stonebraker, J.R., Teed, J., Alb, J.G., Magnuson, T.R., O’Neal, W., and Milgram, S.L. (2006). Defects in yolk sac vasculogenesis, chorioallantoic fusion, and embryonic axis elongation in mice with targeted disruption of Yap65. Mol Cell Biol 26, 77-87.

Nishioka, N., Inoue, K., Adachi, K., Kiyonari, H., Ota, M., Ralston, A., Yabuta, N., Hirahara, S., Stephenson, R.O., Ogonuki, N., et al. (2009). The Hippo signaling pathway components Lats and Yap pattern Tead4 activity to distinguish mouse trophectoderm from inner cell mass. Dev Cell 16, 398-410.

Nishioka, N., Yamamoto, S., Kiyonari, H., Sato, H., Sawada, A., Ota, M., Nakao, K., and Sasaki, H. (2008). Tead4 is required for specification of trophectoderm in pre-implantation mouse embryos. Mech Dev 125, 270-283.

Nowee, M.E., Snijders, A.M., Rockx, D.A., de Wit, R.M., Kosma, V.M., Hämäläinen, K., Schouten, J.P., Verheijen, R.H., van Diest, P.J., Albertson, D.G., et al. (2007). DNA profiling of primary serous ovarian and fallopian tube carcinomas with array comparative genomic hybridization and multiplex ligation-dependent probe amplification. J Pathol 213, 46-55.

Oh, H., and Irvine, K.D. (2008). In vivo regulation of Yorkie phosphorylation and localization. Development 135, 1081-1088.

Oh, H., and Irvine, K.D. (2009). In vivo analysis of Yorkie phosphorylation sites. Oncogene 28, 1916-1927.

Oh, H., Reddy, B.V., and Irvine, K.D. (2009). Phosphorylationindependent repression of Yorkie in Fat-Hippo signaling. Dev Biol 335, 188-197.

Ohnishi, S., Güntert, P., Koshiba, S., Tomizawa, T., Akasaka, R., Tochio, N., Sato, M., Inoue, M., Harada, T., Watanabe, S., et al. (2007). Solution structure of an atypical WW domain in a novel beta-clam-like dimeric form. FEBS Lett 581, 462-468.

Omerovic, J., Puggioni, E.M., Napoletano, S., Visco, V., Fraioli, R., Frati, L., Gulino, A., and Alimandi, M. (2004). Ligand-regulated association of ErbB-4 to the transcriptional co-activator YAP65 controls transcription at the nuclear level. Exp Cell Res 294, $469-479$.

Ota, M., and Sasaki, H. (2008). Mammalian Tead proteins regulate cell proliferation and contact inhibition as transcriptional mediators of Hippo signaling. Development 135, 40594069.

Overholtzer, M., Zhang, J., Smolen, G.A., Muir, B., Li, W., Sgroi, D.C., Deng, C.X., Brugge, J.S., and Haber, D.A. (2006). Transforming properties of YAP, a candidate oncogene on the chromosome 11q22 amplicon. Proc Natl Acad Sci U S A 103, 12405-12410.

Pan, D. (2007). Hippo signaling in organ size control. Genes Dev 21, 886-897.

Pantalacci, S., Tapon, N., and Léopold, P. (2003). The Salvador 
partner Hippo promotes apoptosis and cell-cycle exit in Drosophila. Nat Cell Biol 5, 921-927.

Polyak, K., and Weinberg, R.A. (2009). Transitions between epithelial and mesenchymal states: acquisition of malignant and stem cell traits. Nat Rev Cancer 9, 265-273.

Praskova, M., Xia, F., and Avruch, J. (2008). MOBKL1A/MOBKL1B phosphorylation by MST1 and MST2 inhibits cell proliferation. Curr Biol 18, 311-321.

Ren, F., Zhang, L., and Jiang, J. (2010). Hippo signaling regulates Yorkie nuclear localization and activity through 14-3-3 dependent and independent mechanisms. Dev Biol 337, 303-312.

Richardson, A.L., Wang, Z.C., De Nicolo, A., Lu, X., Brown, M., Miron, A., Liao, X., Iglehart, J.D., Livingston, D.M., and Ganesan, S. (2006). X chromosomal abnormalities in basal-like human breast cancer. Cancer Cell 9, 121-132.

Rodriguez, S., Khabir, A., Keryer, C., Perrot, C., Drira, M., Ghorbel, A., Jlidi, R., Bernheim, A., Valent, A., and Busson, P. (2005). Conventional and array-based comparative genomic hybridization analysis of nasopharyngeal carcinomas from the Mediterranean area. Cancer Genet Cytogenet 157, 140-147.

Sawada, A., Kiyonari, H., Ukita, K., Nishioka, N., Imuta, Y., and Sasaki, H. (2008). Redundant roles of Tead1 and Tead2 in notochord development and the regulation of cell proliferation and survival. Mol Cell Biol 28, 3177-3189.

Scheel, H., and Hofmann, K. (2003). A novel interaction motif, SARAH, connects three classes of tumor suppressor. Curr Biol 13, R899-R900.

Shimizu, T., Seto, A., Maita, N., Hamada, K., Tsukita, S., Tsukita, S., and Hakoshima, T. (2002). Structural basis for neurofibromatosis type 2. Crystal structure of the merlin FERM domain. J Biol Chem 277, 10332-10336.

Silva, E., Tsatskis, Y., Gardano, L., Tapon, N., and McNeill, H. (2006). The tumor-suppressor gene fat controls tissue growth upstream of expanded in the hippo signaling pathway. Curr Biol 16, 2081-2089.

Singh, B., Gogineni, S.K., Sacks, P.G., Shaha, A.R., Shah, J.P., Stoffel, A., and Rao, P.H. (2001). Molecular cytogenetic characterization of head and neck squamous cell carcinoma and refinement of 3q amplification. Cancer Res 61, 4506-4513.

Skotheim, R.I., Autio, R., Lind, G.E., Kraggerud, S.M., Andrews, P.W., Monni, O., Kallioniemi, O., and Lothe, R.A. (2006). Novel genomic aberrations in testicular germ cell tumors by array-CGH, and associated gene expression changes. Cell Oncol 28, 315-326.

Snijders, A.M., Schmidt, B.L., Fridlyand, J., Dekker, N., Pinkel, D., Jordan, R.C., and Albertson, D.G. (2005). Rare amplicons implicate frequent deregulation of cell fate specification pathways in oral squamous cell carcinoma. Oncogene 24, 4232-4242.

Stavridi, E.S., Harris, K.G., Huyen, Y., Bothos, J., Verwoerd, P.M., Stayrook, S.E., Pavletich, N.P., Jeffrey, P.D., and Luca, F.C. (2003). Crystal structure of a human Mob1 protein: toward understanding Mob-regulated cell cycle pathways. Structure 11, 1163-1170.

Steinhardt, A.A., Gayyed, M.F., Klein, A.P., Dong, J., Maitra, A., Pan, D., Montgomery, E.A., and Anders, R.A. (2008). Expression of Yes-associated protein in common solid tumors. Hum Pathol 39, 1582-1589.

Strano, S., Munarriz, E., Rossi, M., Castagnoli, L., Shaul, Y., Sacchi, A., Oren, M., Sudol, M., Cesareni, G., and Blandino, G. (2001). Physical interaction with Yes-associated protein enhances p73 transcriptional activity. J Biol Chem 276, 15164-15173.
Tapon, N., Harvey, K.F., Bell, D.W., Wahrer, D.C., Schiripo, T.A., Haber, D.A., and Hariharan, I.K. (2002). salvador Promotes both cell cycle exit and apoptosis in Drosophila and is mutated in human cancer cell lines. Cell 110, 467-478.

Tian, W., Yu, J., Tomchick, D.R., Pan, D., and Luo, X. (2010). Structural and functional analysis of the YAP-binding domain of human TEAD2. Proc Natl Acad Sci U S A 107, 7293-7298.

Tyler, D.M., and Baker, N.E. (2007). Expanded and fat regulate growth and differentiation in the Drosophila eye through multiple signaling pathways. Dev Biol 305, 187-201.

Udan, R.S., Kango-Singh, M., Nolo, R., Tao, C., and Halder, G. (2003). Hippo promotes proliferation arrest and apoptosis in the Salvador/Warts pathway. Nat Cell Biol 5, 914-920.

Varelas, X., Miller, B.W., Sopko, R., Song, S., Gregorieff, A., Fellouse, F.A., Sakuma, R., Pawson, T., Hunziker, W., McNeill, H., et al. (2010). The Hippo pathway regulates Wnt/beta-catenin signaling. Dev Cell 18, 579-591.

Vassilev, A., Kaneko, K.J., Shu, H., Zhao, Y., and DePamphilis, M.L. (2001). TEAD/TEF transcription factors utilize the activation domain of YAP65, a Src/Yes-associated protein localized in the cytoplasm. Genes Dev 15, 1229-1241.

Wang, Y., Dong, Q., Zhang, Q., Li, Z., Wang, E., and Qiu, X. (2010). Overexpression of yes-associated protein contributes to progression and poor prognosis of non-small-cell lung cancer. Cancer Sci 101, 1279-1285.

Weber, R.G., Sabel, M., Reifenberger, J., Sommer, C., Oberstrass, J., Reifenberger, G., Kiessling, M., and Cremer, T. (1996). Characterization of genomic alterations associated with glioma progression by comparative genomic hybridization. Oncogene 13, 983-994.

Willecke, M., Hamaratoglu, F., Kango-Singh, M., Udan, R., Chen, C. L., Tao, C., Zhang, X., and Halder, G. (2006). The fat cadherin acts through the hippo tumor-suppressor pathway to regulate tissue size. Curr Biol 16, 2090-2100.

Wu, S., Huang, J., Dong, J., and Pan, D. (2003). hippo encodes a Ste20 family protein kinase that restricts cell proliferation and promotes apoptosis in conjunction with salvador and warts. Cell $114,445-456$.

Wu, S., Liu, Y., Zheng, Y., Dong, J., and Pan, D. (2008). The TEAD/ TEF family protein Scalloped mediates transcriptional output of the Hippo growth-regulatory pathway. Dev Cell 14, 388-398.

Xu, T., Wang, W., Zhang, S., Stewart, R.A., and Yu, W. (1995). Identifying tumor suppressors in genetic mosaics: the Drosophila lats gene encodes a putative protein kinase. Development 121, 1053-1063.

Yagi, R., Chen, L.F., Shigesada, K., Murakami, Y., and Ito, Y. (1999). A WW domain-containing yes-associated protein (YAP) is a novel transcriptional co-activator. EMBO J 18, 2551-2562.

Yagi, R., Kohn, M.J., Karavanova, I., Kaneko, K.J., Vullhorst, D., DePamphilis, M.L., and Buonanno, A. (2007). Transcription factor TEAD4 specifies the trophectoderm lineage at the beginning of mammalian development. Development 134, 3827-3836.

Yockey, C.E., Smith, G., Izumo, S., and Shimizu, N. (1996). cDNA cloning and characterization of murine transcriptional enhancer factor-1-related protein 1, a transcription factor that binds to the MCAT motif. J Biol Chem 271, 3727-3736.

Yokoyama, T., Osada, H., Murakami, H., Tatematsu, Y., Taniguchi, T., Kondo, Y., Yatabe, Y., Hasegawa, Y., Shimokata, K., Horio, Y., et al. (2008). YAP1 is involved in mesothelioma development and 
negatively regulated by Merlin through phosphorylation. Carcinogenesis 29, 2139-2146.

Yu, J., Zheng, Y., Dong, J., Klusza, S., Deng, W.M., and Pan, D. (2010). Kibra functions as a tumor suppressor protein that regulates Hippo signaling in conjunction with Merlin and Expanded. Dev Cell 18, 288-299.

Zender, L., Spector, M.S., Xue, W., Flemming, P., Cordon-Cardo, C., Silke, J., Fan, S.T., Luk, J.M., Wigler, M., Hannon, G.J., et al. (2006). Identification and validation of oncogenes in liver cancer using an integrative oncogenomic approach. Cell 125, 1253-1267.

Zeng, Q., and Hong, W. (2008). The emerging role of the hippo pathway in cell contact inhibition, organ size control, and cancer development in mammals. Cancer Cell 13, 188-192.

Zhang, H., Liu, C.Y., Zha, Z.Y., Zhao, B., Yao, J., Zhao, S., Xiong, Y., Lei, Q.Y., and Guan, K.L. (2009). TEAD transcription factors mediate the function of TAZ in cell growth and epithelial-mesenchymal transition. J Biol Chem 284, 13355-13362.

Zhang, L., Ren, F., Zhang, Q., Chen, Y., Wang, B., and Jiang, J. (2008). The TEAD/TEF family of transcription factor Scalloped mediates Hippo signaling in organ size control. Dev Cell 14, 377-387.

Zhao, B., Li, L., Lei, Q., and Guan, K.L. (2010). The Hippo-YAP pathway in organ size control and tumorigenesis: an updated version. Genes Dev 24, 862-874.

Zhao, B., Wei, X., Li, W., Udan, R.S., Yang, Q., Kim, J., Xie, J., Ikenoue, T., Yu, J., Li, L., et al. (2007). Inactivation of YAP oncoprotein by the Hippo pathway is involved in cell contact inhibition and tissue growth control. Genes Dev 21, 2747-2761.

Zhao, B., Ye, X., Yu, J., Li, L., Li, W., Li, S., Yu, J., Lin, J.D., Wang, C. Y., Chinnaiyan, A.M., et al. (2008). TEAD mediates YAPdependent gene induction and growth control. Genes Dev 22, 1962-1971. 